\title{
Ulnar Shortening Osteotomy After Distal Radius Fracture Malunion: Review of Literature
}

\author{
Katarina Barbaric ${ }^{*}$, , Gordan Rujevcan ${ }^{2}$, Marko Labas ${ }^{3}$, Domagoj Delimar ${ }^{4}$ and Goran Bicanic ${ }^{4}$ \\ ${ }^{I}$ Department of Orthopaedic Surgery, Clinical Hospital Centre Zagreb, Salata 6, 10000, Zagreb, Croatia \\ ${ }^{2}$ Department of Orthopaedic Surgery, General Hospital "Dr. Ivo Pedisic" Sisak, J. J. Strossmayera 59, 44000 Sisak, \\ Croatia \\ ${ }^{3}$ Department of Orthopaedic Surgery and Traumatology, General Hospital Varazdin, Ivana Mestrovica 2, 42000 \\ Varazdin, Croatia \\ ${ }^{4}$ Department of Orthopaedic Surgery, University of Zagreb School of Medicine, Clinical Hospital Centre Zagreb, Salata \\ 6-7, 10000 Zagreb, Croatia
}

\begin{abstract}
Malunion of distal radius fracture is often complicated with shortening of the radius with disturbed radio- ulnar variance, frequently associated with lesions of triangular fibrocartilage complex and instability of the distal radioulnar joint. Positive ulnar variance may result in wrist pain located in ulnar part of the joint, limited ulnar deviation and forearm rotation with development of degenerative changes due to the overloading that occurs between the ulnar head and corresponding carpus. Ulnar shortening osteotomy (USO) is the standard procedure for correcting positive ulnar variance. Goal of this procedure is to minimize the symptoms by restoring the neutral radio - ulnar variance. In this paper we present a variety of surgical techniques available for ulnar shorthening osteotomy, their advantages and drawbacks. Methods of ulnar shortening osteotomies are divided into intraarticular and extraarticular. Intraarticular method of ulnar shortening can be performed arthroscopically or through open approach. Extraarticular methods include subcapital osteotomy and osteotomy of ulnar diaphysis, which depending on shape can be transverse, oblique, and step cut. All of those osteotomies can be performed along wrist arthroscopy in order to dispose and treat possibly existing triangular fibrocartilage complex injuries. At the end we described surgical procedures that can be done in case of ulnar shorthening osteotomy failure.
\end{abstract}

Keywords: malunion, techniques, ulna, ulnar shorthening osteotomy, ulnar variance, wrist.

\section{INTRODUCTION}

Distal radius fracture is one of the most common fractures accounting for around $25 \%$ of fractures in the pediatric population and up to $18 \%$ of all fractures in the elderly age [1]. Malunion of distal radius fracture often results in radial shortening as the main deformity, with disturbed radio-ulnar variance, quite often associated with lesions of triangular fibrocartilage complex (TFCC) and instability of the distal radioulnar joint (DRUJ). Radio-ulnar variance is defined as the difference in length between the distal ulnar corner of the radius and the most distal aspect of the dome of the ulnar head. Positive ulnar variance means that the dome of the distal ulna is more distal than the ulnar corner of the distal radius [2] (Fig. 1). This positive variance may lead to ulnar sided wrist pain, limited ulnar deviation and forearm rotation with development of degenerative changes due to the overloading that occurs between the ulnar head and the ulnar carpus. Impaction of the ulnar head

*Address correspondence to this author at the Department of Orthopaedic Surgery, Clinical Hospital Centre Zagreb, Salata 6, 10000, Zagreb, Croatia; Tel: +385 12368 986; Fax: +385 12379 913;

E-mail: katarina.barbaric@hotmail.com

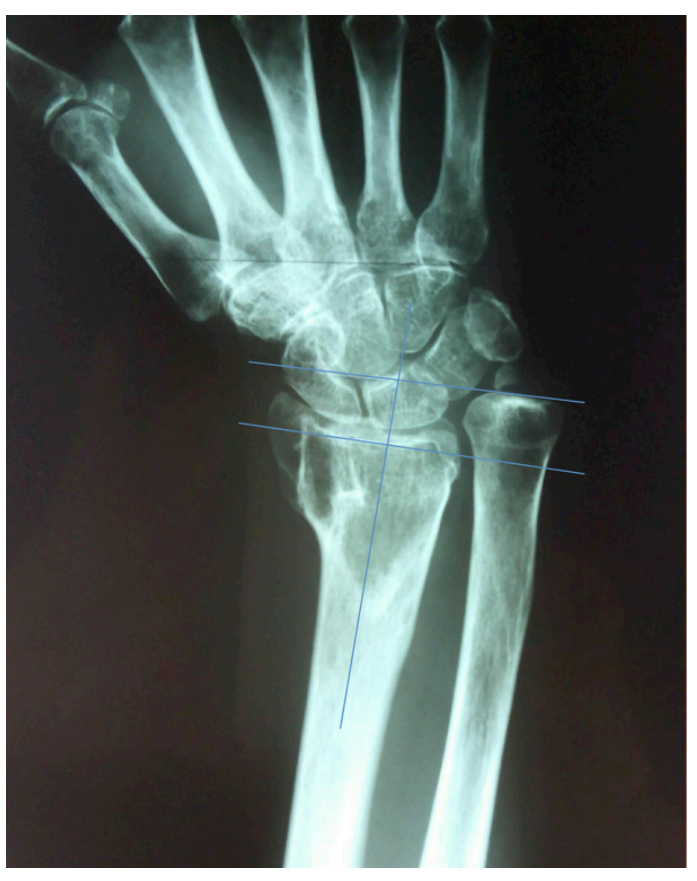

Fig. (1). Radio-ulnar variance measurement. 
against the carpus produces degenerative tears of the TFCC, chondromalacia of the ulnar head, lunate, or triquetrum; attenuation and tears of the triquetrolunate ligament; and, finally, ulnocarpal osteoarthritis. Instability of the DRUJ occurs due to loss of ligament support after rupture or avulsion of the TFCC. Damage of secondary joint stabilizers (the capsular ligaments, the sheath of the extensor carpi ulnaris, the interosseous membrane, and the pronator quadratus) or extra-articular and intra-articular osseous disruption of the joint surface may increase the degree of laxity [3].

Although corrective osteotomy of the distal part of the radius restores anatomical relations between distal radius and ulna it may often be technically difficult procedure $[4,5]$. On the other hand, in some cases with minimal radial angulation that kind of procedure is even unnecessary. That is why ulnar shortening osteotomy (USO) has become the standard for correcting positive ulnar variance $[6,7]$. Goal of the shortening procedure is to relieve the symptoms by reestablishing a neutral radio-ulnar variance (Fig. 2). Many authors described different options for this procedure in order to achieve the best possible functional results. In this paper we present a variety of surgical techniques available for USO, their advantages and drawbacks.

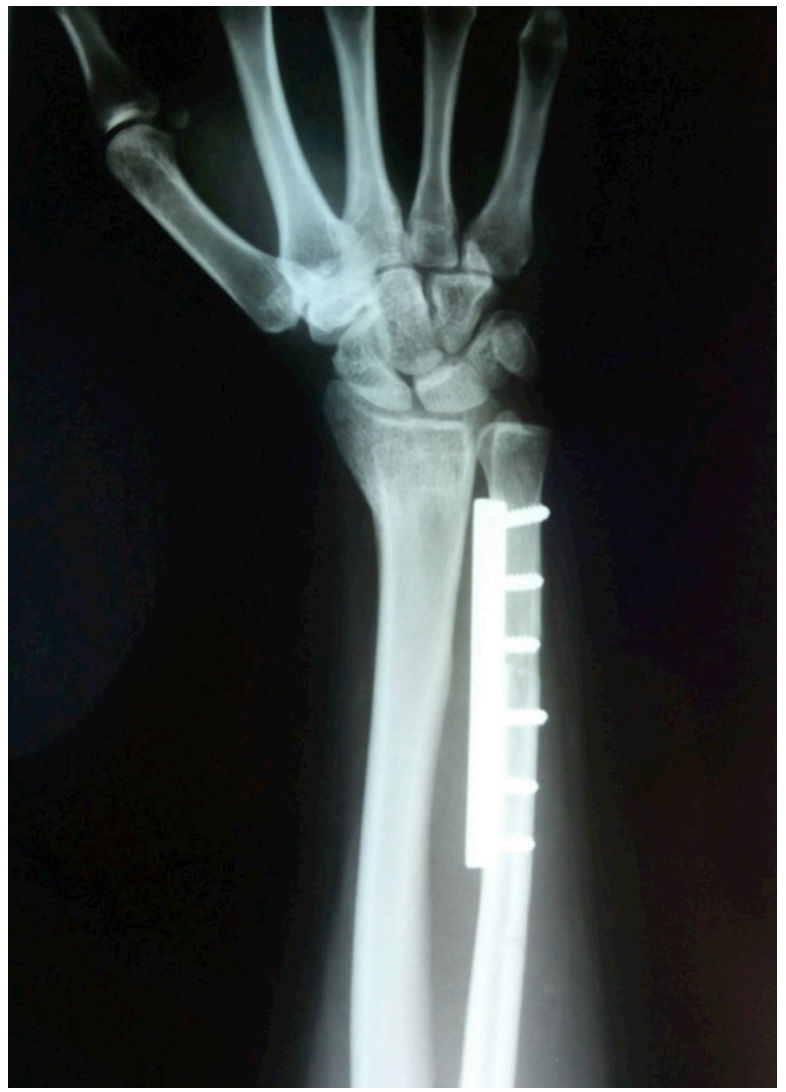

Fig. (2). Radiogram showing postoperative neutral radio-ulnar variance achieved.

\section{ANATOMY AND BIOMECHANICS}

For normal function of the wrist and forearm extremely important are anatomic relationships of the radius and ulna, in the proximal and distal radioulnar joint and relationships both distal articular surfaces with the carpal bones.
Articulation between proximal carpal row and distal radius and TFCC on the other side is C-shaped. Normally, the radius and ulna are almost the same length. Changes in the length of the ulna relative to the length of the radius, designated ulnar variance, alter the distribution of compressive forces across the wrist. Ulnar variance is measured on the anteroposterior radiograms of the wrist by extending a line along the distal articular surface of the radius toward the ulna and measuring the distance from this line to the distal ulna [8]. Altered relationships between the radius and ulna like in distal radius malunion followed by radius shortening and consequently longer ulna lead to compression of the ulnar side of the carpus and changes in the distal radioulnar joint. The DRUJ is a distal articulation in the biarticulate rotational arrangement of the forearm that allows pronation and supination. In DRUJ shallow sigmoid notch of the ulnar aspect of the distal radius articulates with the circular head of the ulna. The contact between radius and ulna is relatively small which provides minimal stability of osseous anatomy. The radius of curvature of the ulnar head does not equal one of the sigmoid notch. In the extremes of pronation-supination, less than $10 \%$ of the ulnar head may be in contact with the notch. Because its skeletal architecture imparts minimal stability to the DRUJ, the main stability is achieved by soft tissue through extrinsic (extracapsular) and intrinsic (intracapsular) stabilizers [9]. Extrinsic stability is provided principally by dynamic tensioning of the extensor carpi ulnaris as its tendon crosses the distal head of the ulna, the semirigid sixth dorsal compartment itself, constraining the extensor carpi ulnaris tendon, superficial and deep heads of the musculus pronator quadratus, and the interosseous membrane of the forearm [10-13]. The rotational stability provided by extrinsic stabilizers is of secondary importance compared with the more biomechanically effective intrinsic radioulnar stabilizers $[9,14,15]$. The intrinsic stabilizer is TFCC. The TFCC include the triangular fibrocartilage disc and dorsal and palmar radioulnar fibers arising from the medial border of the distal radius and insert on the ulna at two separate and distinct sites forming superficial and deep segment. Superfical segment (ligamentum superficiale) attaches to ulnar styloid, while deep segment (ligamentum subcruentum) attaches to the fovea at the base of ulnar styloid. Ligamentum subcruentum is biomechanically more important than ligamentum superficiale in providing stability of DRUJ. The articular disk is primarily responsible for load transmission from the medial carpus to the forearm, particularly with the hand-forearm unit in ulnar deviation. The relationships between the distal radius and ulna and ulnar carpus are precise, and even minor modification in these relationships leads to significant load changes and resultant pain syndromes. When positive ulnar variance exceeds a few millimetres, additional limitations of wrist flexion-extension as well as radial-ulnar deviation movements can occur. Ekenstam et al. have demonstrated that in neutral position of the wrist, $84 \%$ of hand load is transferred to the radius and only $16 \%$ is transferred through the central articular disc of the TFCC [14]. With ulnar deviation of the hand principal axis of load bearing shifts medially, placing more load on the articular disc and the pole of the distal ulna. Rotational movements during pronation and supination of the forearm produce axial loading and yield a "drilling- like" effect of ulna at the ulnar side of the 
carpus, which can cause degenerative changes [16]. Palmer and Werner showed that loads through the distal ulna can change and are directly related to ulnar variance. Increasing the ulnar variance to a positive $2.5 \mathrm{~mm}$ increases ulnar load to $42 \%$ ( $18.4 \%$ to $41.9 \%)$ and shortening of the ulna by 2.5 $\mathrm{mm}$ decreased axial load at distal ulna to $4.3 \%$ [17]. These results suggest that the biomechanics of the wrist joint can be dramatically altered with relatively small changes in ulnar length. Every change of ideal anatomical relationships in the wrist will lead to significant changes in the load transfer in the joint resulting with pain and function impairment and with time severe degenerative changes of the wrist.

\section{METHODS OF ULNAR SHORTENING}

Malunion of distal radius fracture often causes discrepancy in the wrist with a long ulna relative to the radius manifesting with ulnar wrist pain, swelling, and limitation of wrist motion. With positive ulnar variance, the normal load on the ulnar carpus of approximately $18 \%$ increases up to $65 \%$ [18]. That leads to a degenerative change of the ulnocarpal joint and DRUJ.

The importance of this problem was noticed and published back in 1923. by Hoag [19] in his case report of three patients with post-traumatic shortening of the radius. He described the resection of the distal ulna as the method of choice in the treatment of these patients in order to reduce compression on the ulnar carpus. Also in all patients he resected the TFCC because it was ruptured. Patients were immediately started with physical therapy and had satisfactory range of motion and grip strength.

The first ulnar-shortening osteotomy was described by Milch in 1941 [20]. He preformed this procedure on a 17year-old patient who developed a painful ulnar-positive variance wrist due to distal radius malunion. Milch's technique included resection of a portion of the ulnar shaft with wire fixation at the osteotomy site. Since this initial description, numerous authors have described various osteotomy types, including transverse, oblique and step cut. Several commercially available systems have been developed to facilitate bony contact, compression, and rigid fixation at the osteotomy site. Also, methods of intraarticular ulnar shortening have been developed, especially with development of the wrist arthroscopy.

The gold standard treatment, distal ulnar shortening osteotomy addresses the positive ulnar variance and therefore relieves the excessive load on the ulnocarpal joint [21]. There are numerous reports about the technique, different implants and results [22, 23]. All of these studies show promising results with a low complication rate of nonunions, neural lesions, etc. Nishiwaki et al. [24] did a biomechanical cadaveric study in which they stated that the amount of ulnar shortening should be carefully planned to avoid excessive pressure at the DRUJ because the greater the amount of ulnar shortening, the higher is the peak pressure at the DRUJ. They stated that a shortening of up to $3 \mathrm{~mm}$ may be appropriate to stabilize the DRUJ. Arimitsu et al. [25] did a cadaveric biomechanical study about the importance of the distal interosseus membrane on the distal radioulnar joint in USO and they concluded that distal radioulnar joint laxity was decreased by the increased tensioning of the distal interosseous membrane accompanying ulnar shortening. An osteotomy proximal to the ulnar attachment of the distal interosseous membrane should improve distal radioulnar joint stability. Typically, approximately $40 \%$ of distal interosseous membranes have a distal oblique bundle. If so, an enhanced distal radioulnar joint-stabilizing effect can be expected; if not, additional or alternative procedures may be considered when instability of the distal radioulnar joint remains a concern. For patients with ulnar impaction and a stable distal radioulnar joint, an osteotomy performed distal to the ulnar attachment of the distal interosseous membrane, or sectioning of the distal interosseous membrane when it is thick, may allow better healing of the osteotomy site. It is also important to keep in mind the technical aspects of the instruments used. Firoozbakhsh et al. [26] found that the temperature rise for the double thickness saw blade was $14 \%$ higher than the single, and $23 \%$ higher for the triple thickness blade. They also found the speed of the cut to be a significant factor. The higher the speed, the lower the temperature rise. They propose that complications following USO may be secondary to excess heat generation. Also, there are few possible complications following USO including extensor carpi ulnaris tendinitis from hardware irritation, delayed union and nonunion of the osteotomy. The main complication of ulnar shortening osteotomy is a high rate of nonunion, from 10 to $21 \%$ [27-29]. The oblique osteotomy increases the surface area up to $40 \%$ and therefore increases the potential for union [30]. Chen et al. [31] had no cases of nonunion using oblique osteotomy and compression device. Kitzinger et al. [32] report no cases of nonunion using oblique osteotomy without a compression device. On the other hand Ashan et al. [33] published a rate of $7 \%$ nonunions, despite the use of a compression device. Because of all of the above, in the desire to achieve the best possible results different authors have developed variety of USO techniques (Fig. 3).

Today, methods of ulnar shortening can be divided into intraarticular and extraarticular (Table 1). Intraarticular method of ulnar shortening, so called „wafer procedure“ can be performed arthroscopically or through open approach. Extraarticular methods include subcapital osteotomy and osteotomy of ulnar diaphysis. Depending on shape diaphiseal osteotomies are divided on transverse, oblique, and step cut osteotomies. Some authors suggest performing wrist arthroscopy along ulnar osteotomy in order to dispose and treat possibly existing TFCC injuries. In case of failure of those surgical procedures or the impossibility of their performance, there are few available options: distal ulna resection (Darrach procedure), Sauvé-Kapandji procedure, and the hemi-resection interpositional arthroplasty.

\section{EXTRAARTICULAR OSTEOTOMY}

Advantage of extraarticular USO is that it preserves the mechanical integrity of the distal radioulnar joint. Still, there are some considerations regarding the technique, preoperative planning and materials used. There are two main types of extraarticular osteotomies: diaphyseal and subcapital osteotomies. 


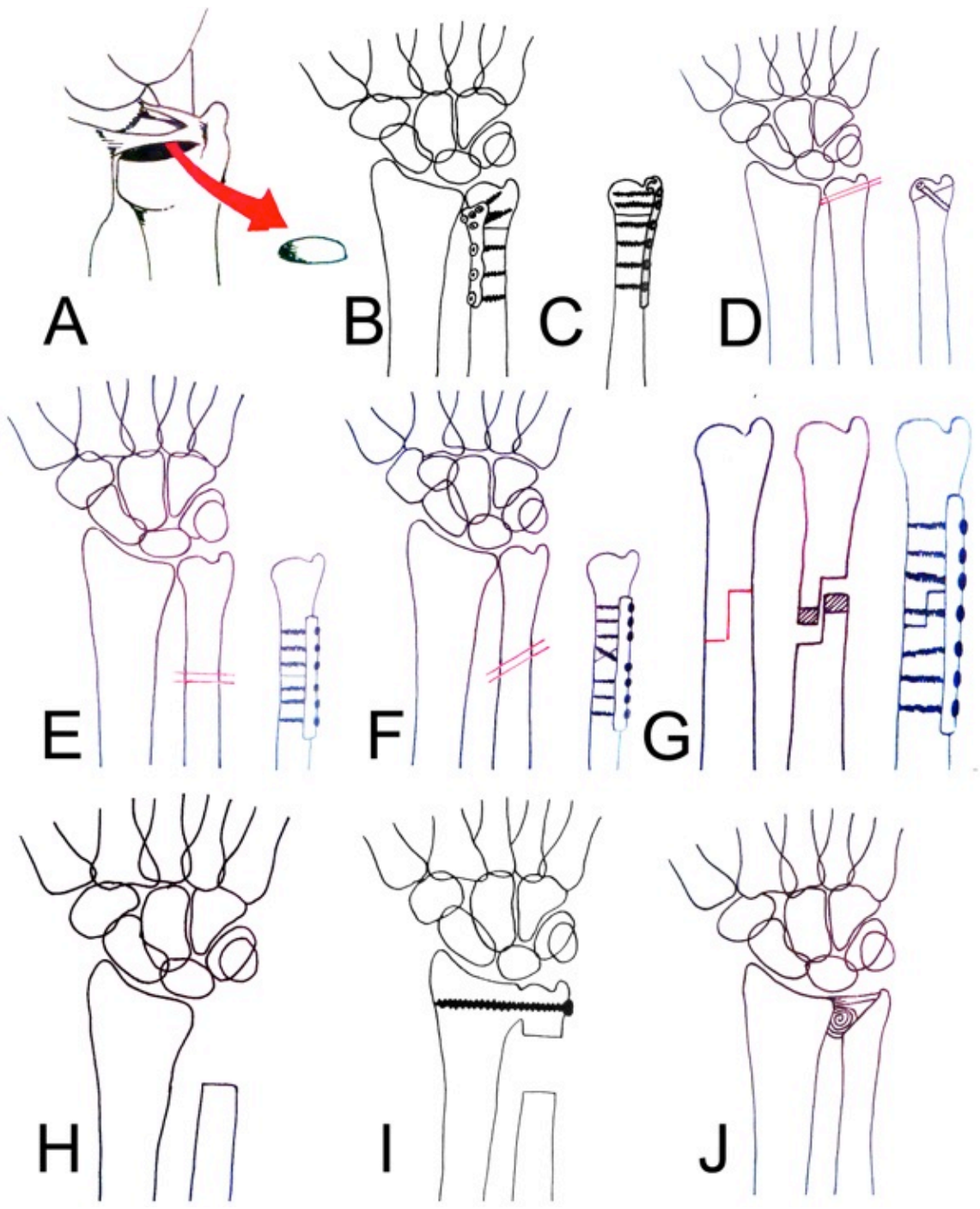

Fig. (3). Methods of ulnar shortening. A) Wafer procedure; B) Subcapital osteotomy- fixation with a locking plate; C) Distal osteotomy with screw fixation; D) Diaphyiseal transverse osteotomy; E) Diaphyseal oblique osteotomy; F) Diaphyseal step-cut osteotomy; G) Darrach procedure; H) Sauvé- Kapandji procedure; I) Hemiresection- interpositional arthroplasty.

\section{Dyaphiseal Osteotomies}

Diaphyseal osteotomies can be divided in three subgroups: transverse, oblique and step cut osteotomies of the ulna. Although they are all still used, reports have shown that oblique osteotomies have a faster healing rate, a lower nonunion rate, when compared to transverse osteotomies [34-36]. Rayhack et al. [36] related that statement to increased surface areas in oblique osteotomies. Some authors use a specially designed device for the osteotomy while the others use „free hand" technique. The most commonly used fixation device is the AO dynamic compression plate [31].

The plate fixation of transverse osteotomy of the ulna was first described by Cantero in 1974 [37]. Transverse osteotomies were later used by many authors with some variations and new techniques and materials. Tatabe et al. [38] used a transverse osteotomy of the ulna fixed with a 3.5 $\mathrm{mm}$ locking compression plate. The contralateral side served as the reference for the length of shortening. In retrospective study including sixteen patients they have found a significant improvement in range of motion postoperatively.
Complications were rare, with no patients showing ulnar non-union. According to literature, malalignment with respect to ulnar inclination and the sigmoid notch has been considered a relative contraindication for USO, because it might cause degenerative changes in the DRUJ [39-41]. Although spur formation occurred in two-thirds of patients in this study, clinical results did not correlate with bony spur formation at the DRUJ. Authors point out advantage of USO in its simplicity and report that the risk of complications in the form of nonunion, delayed union and irritation by the implant could be low if procedure is properly performed. They recommend USO for the surgical treatment of UCA after malunited distal radius fracture, for which the main deformity is moderate radial shortening with a normal radial inclination. In the presence of severe radial shortening and decreased radial inclination, they suggest combined corrective osteotomy of the distal radius. Lautenbach et al. [42] stated that a transverse osteotomy using a 7 hole LC-DC plate and a compression device through an ulnodorsal approach is a simple technique with a low complication rate. They perform it through the incision placed on the distal 
Table 1. Surgical treatment options for positive ulnar variance following radial fracture malunion.

\begin{tabular}{|c|c|c|c|}
\hline \multirow{3}{*}{ Intraarticular USO } & $\begin{array}{l}\text { Closing wedge } \\
\text { osteotomy }\end{array}$ & & $\begin{array}{l}\text { An arthrotomy of the DRUJ, osteotomy performed proximal } \\
\text { to the articular cartilage. A closing wedge osteotomy at the } \\
\text { metaphysis of the distal ulna. Fixation of the osteotomy with } \\
\text { cannulated screws placed from distal to proximal, proximal to } \\
\text { the TFC, through the nonarticular pole of the ulna, avoiding } \\
\text { the articular seat. }\end{array}$ \\
\hline & \multirow[b]{2}{*}{ Wafer procedure } & Open & \multirow{2}{*}{$\begin{array}{c}\text { Removing of distal } 2-4 \mathrm{~mm} \text { of ulnar head with an osteotome, } \\
\text { including articular cartilage and subchondral bone while } \\
\text { preserving the ulnar styloid process and all TFCC } \\
\text { attachments. The majority of the cartilage articulating with } \\
\text { the sigmoid notch of the radius is retained to preserve DRUJ } \\
\text { function. Débridement or repair of the TFCC is also } \\
\text { performed. }\end{array}$} \\
\hline & & Arthroscopic & \\
\hline \multirow{5}{*}{ Extraarticular USO } & \multirow[b]{2}{*}{ Subcapital } & Oblique & \multirow{2}{*}{$\begin{array}{l}\text { Newer implants that include distal locking capabilities in } \\
\text { smaller sized plates permit the osteotomy site to be moved to } \\
\text { the subcapital region of the ulna where short healing times } \\
\text { through cancellous bone can reliably be achieved. Cut } \\
\text { through cancellous bone, proximal to the joint capsule of the } \\
\text { DRUJ. }\end{array}$} \\
\hline & & Transverse & \\
\hline & \multirow{3}{*}{ Diaphyseal } & $\begin{array}{l}\text { Oblique (with/without } \\
\text { arthroscopy) }\end{array}$ & $\begin{array}{l}\text { Two parallel oblique cuts are made followed by removal of } \\
\text { the cut bone. Compression with/without compression device. } \\
\text { A lag screw is placed through the osteotomy site followed by } \\
\text { plate fixation. }\end{array}$ \\
\hline & & $\begin{array}{l}\text { Transverse (woth/without } \\
\text { arthroscopy) }\end{array}$ & $\begin{array}{l}\text { Two parallel transverse cuts after which the cut bone is } \\
\text { removed. Compression with/without a compression device } \\
\text { followed by plate fixation. }\end{array}$ \\
\hline & & Step-cut & $\begin{array}{l}\text { The long arm is cut in the coronal plane parallel to the long } \\
\text { axis of the ulna. The } 2 \text { short arms are cut perpendciular to the } \\
\text { first cut. The bone is removed by cutting parallel to the short } \\
\text { arms of the osteotomy and the osteotomy is then reduced after } \\
\text { which a lag screw is placed followed by plate fixation. }\end{array}$ \\
\hline \multirow{4}{*}{ Other procedures } & Darrach procedure & & Resection of the ulna head. \\
\hline & $\begin{array}{l}\text { Sauvé- Kapandji } \\
\text { procedure }\end{array}$ & & $\begin{array}{l}\text { Fusion of the DRUJ with resection of bone just proximal to } \\
\text { the fusion site }\end{array}$ \\
\hline & $\begin{array}{l}\text { hemiresection- } \\
\text { interpositional } \\
\text { arthroplasty }\end{array}$ & & $\begin{array}{l}\text { Removing of the damaged joint surface while maintaining a } \\
\text { strong soft tissue connection of the ulna to the carpus and } \\
\text { radius. }\end{array}$ \\
\hline & DRUJ prosthesis & & Aloarthroplasty of the DRUJ. \\
\hline
\end{tabular}

ulnar side of the forearm. After contouring 7-hole $3.5 \mathrm{~mm}$ LC-DC plate, they predrill three distal holes, mark edges and rotation of the osteotomy and perform the osteotomy to achieve a neutral or slightly negative ulnar variance. They do the transverse cut without the use of a cutting device, and load the plate at high tension using the compression device. Authors report that transverse osteotomy using a compression device and an ulnodorsal approach is a simple technique with a low complication rate. They think it is comparable to oblique osteotomy in effectiveness and safety but it has the advantage of requiring less experience on the part of the surgeon because the osteotomy is easier to align. Also, no additional devices or special implants other than the compression device are needed. Wehbé et al. [36] evaluated a technique for ulnar shortening utilizing a transverse osteotomy using the AO small distractor and 2.7- $\mathrm{mm}$ dynamic compression plate. In their 24 patients they had no nonunions and an average time to healing of 9.7 weeks. They did have three delayed unions, but these reportedly healed without incident by 28,34 and 36 weeks.

On the other hand, some authors prefer oblique osteotomies. Rayhack et al. [30], in their study, reported a faster healing time with an oblique osteotomy due to the $40 \%$ increase in bony surface area. Their average time to union was 11 weeks. Chen [31] achieved $100 \%$ bony union in his 18 patients in an average of 6.8 weeks with an oblique osteotomy. Similarly, Kitzinger [32] had a $100 \%$ union rate 
using an oblique osteotomy with a newly developed palmarly placed sliding-hole dynamic compression plate that allows fixation of the ulna before the osteotomy is carried out. Clark et al. [43] used a low profile compression plate designed specifically for an oblique ulnar shortening osteotomy. The system's unique plate design allows for locked fixation of the plate to the bone, prior to osteotomy, to allow for controlled compression at the osteotomy site and has cutting guides that attach directly to the plate. They also state that biomechanical studies have shown that structural stiffness in torsion is clearly greater for oblique osteotomy $[30,34]$. Isaacs et al. [44] used an oblique osteotomy with Kwires to stop malrotaion and the use of cutting guides. A bicortical K- wire was used for the placement of a compression clamp. After compression they used a lag screw. Luria et al. [2] compared systems developed by two manufacturers. The Synthes dynamic compression system which uses a freehand oblique osteotomy and the Synthes distraction device followed by a lag screw and a 3,5 mm 6hole DC plate. The same technique was described by Chen $e t$ al. [31]. The comparison was made with the Trimed ulnarshortening osteotomy (DC) system that utilizes cutting guides and a guide for the lag screw eliminating the need for freehand osteotomies. They believe that the shorter procedure time in the latter procedure is due to Trimed system's technical advantages. Schmidle et al. [45] used a specially designed osteotomy system with saw guides and a setscrew for compression after the osteotomy. Since the osteotomy is oblique they used a lag screw for stabilization.

Darlis et al. [46] described a simple step-cut ulnarshortening osteotomy stabilized with a lag screw and a palmarly placed $3.5-\mathrm{mm}$ neutralization plate. The step-cut osteotomy has its long arm oriented in the coronal plane parallel to the long axis of the ulna. The short arms of the osteotomy are perpendicular to the long axis in the axial plane. The osteotomy, approximately $2 \mathrm{~cm}$ in length, is designed to extend from the third to the fifth holes of the plate, making sure that there is room left for bicortical screw placement in these 2 holes. In most situations a 2- to $4-\mathrm{mm}$ shortening of the ulna is desired, and use of the 7-hole plate as a template ensures that the arms of the step-cut will have sufficient length for lag-screw placement after shortening. The planed osteotomy is outlined on the cortex of the ulna with a marking pen. The long arm of the osteotomy is cut first using an oscillating saw. Care is taken to orient this cut in a medial-to-lateral direction and to cut both cortices. The 2 short arms are cut next in the axial plane perpendicular to the first cut. Next the intended length of shortening is marked on both the proximal and distal osteotomy arms. The bone is removed by cutting parallel to the short arms of the osteotomy and the osteotomy is then reduced. Ulnar deviation of the wrist facilitates approximation of the bone ends. Once reduction is deemed satisfactory it is secured with a reduction clamp across the osteotomy, with care taken to leave room for lag screw placement. A lag screw then is drilled and inserted from dorsal to volar perpendicular to the long arm of the osteotomy. Finally the 7-hole dynamic compression plate is placed on the volar flat surface of the ulna and centered on the osteotomy. Three screws are inserted on each side of the osteotomy in the neutralization mode. The fixation technique can be modified so that compression is applied through the plate as well. They find the latter technique to be more cumbersome. The step-cut osteotomy resulted in solid union in all patients. It provides ample bone-to-bone contact and easier control of rotation, and no special instrumentation is necessary. Stable fixation permitted early mobilization of the wrist and palmar placement of the plate minimized the need for plate removal.

\section{Subcapital Osteotomy}

Ulnar osteotomy has traditionally been performed in the diaphysis and secured with a $3.5 \mathrm{~mm}$ plate. Newer implants that include distal locking capabilities in smaller sized plates permit the osteotomy site to be moved to the subcapital region of the ulna where short healing times through cancellous bone can reliably be achieved. Henry [47] proposed the use of a subcapital ulnar shortening osteotomy and the use of a $2.3 \mathrm{~mm}$ locking plate making sure the intended cut is through cancellous bone, oblique, and proximal to the joint capsule of the DRUJ. That technique should avoid complications related to diaphyseal osteotomy like delayed union, nonunion, and local irritation caused by bulky $3.5 \mathrm{~mm}$ plate. Nunez et al. [48] used a locking compression distal ulna plate which has hooks that are engaged into the tip of the ulnar styloid. Once a satisfactory position of the plate is confirmed using fluoroscopy, distal drill holes are made using a $1.5-\mathrm{mm}$ drill bit. The site of the osteotomy in the metaphysis of the ulna is marked, the plate is removed, and two transverse parallel osteotomies are created just proximal to the DRUJ. After bone removal and positioning of the plate distal locking screws are inserted in the previously drilled holes of the ulnar head. The osteotomy is closed using pointed reduction forceps. A drill guide may be inserted into a locking hole of the plate proximal to the osteotomy and used as a handle to pull the plate along with the distal fragment proximally which may help further reduce and compress the osteotomy. There is a $2.0-\mathrm{mm}$ selftapping cortical screw inserted excentricaly into the oblong hole of the shaft to obtain axial compression of the osteotomy. Authors report that bone healing was achieved in all patients with no hardware-related pain or required further surgery for hardware removal.

\section{EXTRAARTICULAR ULNAR SHORTHENING OSTEOTOMY WITH ARTHROSCOPY}

Some authors perform wrist arthroscopy prior to an ulnar shortening osteotomy.

They concluded that diagnostic arthroscopy should be done prior to an osteotomy in case of TFCC tears or articular disk changes so they can be debrided. Baek et al. [49] proposed using a conventional transverse ulnar shortening osteotomy in combination with arthroscopy if there were cystic changes of the carpus showed on radiographs or degenerative TFCC tears showed on MRI. They used a 6 hole standard compression plate or a one third tubular plate which they placed on volar side. They used a compressive screw and no compressive device. Koh et al. [50] proposed a transverse osteotomy at 5 to $6 \mathrm{~cm}$ proximal to the ulnar styloid with secure fixation with a 5- or 6- hole $3.5 \mathrm{~mm}$ limited contact dynamic compression plate. Diagnostic arthroscopy which also included debridement of the unstable central portion of the articular disk, and cartilage flap, repair 
of the peripheral tear and thermal shrinkage of the articular disk if necessary, were performed before the osteotomy.

As with transverse osteotomies arthroscopy prior to oblique ulnar shortening osteotomy was described, also with debridement of the wrist if needed. The use of arthroscopy prior to oblique USO was described by Kim et al. [51] who compared oblique ulnar shortening osteotomies alone with those following wrist arthroscopy and debridement if needed. They used a 7-hole small dynamic compression plate on the volar side of the ulna and inserted screws in a compression mode. The conclusion was that there is no difference in clinical outcomes between these two methods.

\section{INTRAARTICULAR OSTEOTOMIES}

Wafer procedure is an intraarticular shortening osteotomy which can be done through open approach or arthroscopically. The open wafer procedure was first described by Feldon et al. in 1992 [52]. The procedure includes removing of distal $2-4 \mathrm{~mm}$ of ulnar head with an osteotome, including articular cartilage and subchondral bone while preserving the ulnar styloid process and all TFCC attachments. The majority of the cartilage articulating with the sigmoid notch of the radius is retained to preserve DRUJ function. Debridement or repair of the TFCC is also performed. Advantage of this procedure is that it avoids complications such as nonunions associated with ulnar shortening osteotomy. The main disadvantage is that it involves removing of articular surface of the distal ulna. The procedure is indicated in patients with less then $4 \mathrm{~mm}$ of ulnar-positive variance, if there are no signs of degenerative arthritis or instability of the DRUJ. Good to excellent results have been obtained in $85 \%$ to $100 \%$ of patients with near full recovery of motion and grip strength [52-55]. However according to Constantine et al. [23] in their retrospective comparison study between ulnar shortening osteotomy and wafer procedure, no difference between those two methods was found.

Arthroscopic wafer distal ulna resection was first reported in the 1990. For patients presenting with extensive degenerative TFCC tears, chondromalatia of the ulnar head, or arthritis of the DRUJ [56]. It is indicated in patients with less than $3 \mathrm{~mm}$ of ulnar-positive variance, because more than 2 to $3 \mathrm{~mm}$ is difficult to remove arthroscopically [57, 58]. It is also good method for repairing TFCC tears.

In 2007, Slade and Gillon [59] introduced a unique technique of ulnar shortening. This technique, although intra-articular and requiring an arthrotomy of the DRUJ, is performed proximal to the articular cartilage. The technique uses a closing wedge osteotomy performed at the metaphysis of the distal ulna. Fixation of the osteotomy is achieved using cannulated screws. The screws are placed from distal to proximal, proximal to the TFC, through the nonarticular pole of the ulna, avoiding the articular seat. The advantages of this technique include rapid union because the osteotomy is performed through metaphyseal bone, fixation achieved with low profile implants that do not need removal, early mobilization, preservation of the articular loadbearing portion of the DRUJ, and limited exposure and dissection when compared with open diaphyseal techniques. Potential drawbacks include an intraarticular approach that may lead to capsular scarring and stiffness, hardware-related complications, and potential compromise of the distal fragment leading to non- or malunion. Greenberg et al. [60] in 2013 performed biomechanical analysis of that procedure to investigate the effect of a closing wedge osteotomy at the distal ulnar metaphysis on unloading the ulnar side of the wrist. They concluded that this technique is an alternative to open diaphyseal techniques or methods that damage the articular surface of the distal ulna but emphasized the importance of performing clinical studies to associate the biomechanical results with clinical symptom improvement.

\section{OTHER PROCEDURES}

In case of contraindications for the above mentioned procedures or postoperative complications or persistent DRUJ pain there are a few procedures that can still be used: distal ulnar resection (Darrach), the Sauvé-Kapandji procedure, and the hemi-resection interpositional arthroplasty. These techniques should be reserved as salvage procedures.

Darrach procedure is resection of the ulna head, originally described by Darrach in 1913 [61]. Although some authors suggest that the Darrach procedure provides reliably good long-term subjective and objective results for the treatment of a symptomatic DRUJ after a distal radius fracture. Patients can expect to have excellent forearm range of motion at long-term follow-up. Nearly one-half of patients had dynamic convergence of the DRUJ when stressed radiographically; however, the presence of radiographic dynamic convergence did not influence clinical outcomes [62]. Other authors disagree and say that Darrach's technique is clearly wrought with biomechanical consequences, the most common of which relate to loss of an ulna load-bearing fulcrum at the DRUJ [63].

The first detailed description of a fusion of the DRUJ with resection of bone just proximal to the fusion site was published by Louis Sauvé and Mehmed Kapandji in 1936 [64]. Since then, different authors suggest several modifications of the procedure, but the name "SauvéKapandji procedure" has remained. Shors and Baratz [65] considered that Sauvé-Kapandji procedure after distal radius fracture is indicated in active, high-demand patient with DRUJ arthritis and in cases failed ulnar shortening or ligament reconstruction for DRUJ instability. Contraindication for this procedure is inadequate bone stock at the DRUJ. According to literature this procedure has good postoperative results. Patients have almost normal forearm rotation, mild to moderate pain, unchanged wrist flexionextension, and increased ability to return to work [66-68].

Hemi-resection interpositional arthroplasty procedure was developed by William H. Bowers in 1981 as an integral part of managing the rheumatoid ulnar wrist. The principle of the procedure is to maintain a strong soft tissue connection of the ulna to the carpus and radius while removing the damaged joint surface. Although originally designed primarily for the rheumatoid wrist, this procedure has also been used successfully as a reconstructive option in the setting of distal radial malunion $[69,70]$. In carefully selected cases results are generally good to excellent. On the other hand, in patients with significant ongoing symptoms 
after this procedure, a DRUJ prosthesis can still be utilized with success.

Operative procedures described here should be preceded by careful preoperative planning and followed by specific rehabilitation protocols regarding the technique used.

\section{CONCLUSION}

Ulnar shortening osteotomies are a group of procedures that are performed for symptomatic positive ulnar variance which is often a complication of distal radial fractures. USO is the gold standard for the treatment of such complications. Many authors described different systems and techniques for the osteotomy in order to achieve the best possible functional results. The goal of any ulnar shortening osteotomy is to obtain neutral ulnar variance with good apposition and compression at the osteotomy site. Problems with delayed healing or nonunion after osteotomy complicate the shortening procedure. By performing oblique osteotomy or placing it in the metaphyseal or subcapital region authors try to create better conditions for faster bone healing. Using the newer, smaller implants is a way to reduce the number of complications related to hardware irritation. The introduction of wrist arthroscopy improved the treatment because of the possibility of disposal and treatment of intra-articular damages such as rupture of the TFCC. On the other hand, surgeon's experience with his particular method is maybe more important than the advantages or disadvantages of any described techniques. High satisfaction rate and good functional outcome in our patients suggest that ulnar shortening osteotomy is a valid therapeutic option. But we have to keep in mind that ulnar shortening osteotomies leave the radius angular deformities unchanged. If displacement of the radius is mainly limited to loss of length, then ulnar shortening osteotomy is adequate treatment for ulnarsided wrist pain. In cases with more severe angular displacements of the distal radius we should decide for radial corrective osteotomy of combination of these two procedures.

\section{CONFLICT OF INTEREST}

The authors confirm that this article content has no conflict of interest.

\section{ACKNOWLEDGEMENTS}

Declared none.

\section{REFERENCES}

[1] Nellans KW, Kowalski E, Chung KC. The epidemiology of distal radius fractures. Hand Clin 2012; 28(2): 113-25.

[2] Luria S, Lauder AJ, Trumble TE. Comparison of ulnar-shortening osteotomy with a new Trimed dynamic compression system versus the Synthes dynamic compression system: clinical study. J Hand Surg Am 2008 Nov; 33(9): 1493-7.

[3] Jupiter JB, Fernandez DL. Complications following distal radial fractures. Instr Course Lect 2002; 51: 203-19.

[4] Fernandez DL. Correction of post-traumatic wrist deformity in adults by osteotomy, bone grafting and internal fixation. J Bone Joint Surg Am 1982; 64-8: 1164-78.

[5] Jupiter JB, Ruder J, Roth DA. Computer-generated bone models in the planning of osteotomy of multidirectional distal radius malunions. J Hand Surg Am 1992; 17(3): 406-15.
[6] Fricker R, Pfeiffer KM, Troeger H. Ulnar shortening osteotomy in posttraumatic ulnar impaction syndrome. Arch Orthop Trauma Surg 1996; 115(3-4): 158-61.

[7] Oskam J, Kingma J, Klasen HJ. Ulnar-shortening osteotomy after fracture of the distal radius. Arch Orthop Trauma Sur 1993; 112(4): 198-200.

[8] Loredo RA, Sorge DG, Garcia G. Radiographic evaluation of the wrist: a vanishing art. Semin Roentgenol 2005; 40(3): 248-89.

[9] Kleinman WB. Stability of the distal radioulnar joint: biomechanics, pathophysiology, physical diagnosis, and restoration of function what we have learned in 25 years. J Hand Surg Am 2007; 32(7): 1086-106.

[10] Goldner JL, Hayes MG. Stabilization of the remaining ulna using one-half of the extensor carpi ulnaris tendon after resection of the distal ulna. Orthop Trans 1979; 3: 330-1.

[11] Spinner M, Kaplan EB. Extensor carpi ulnaris. Its relationship to stability of the distal radioulnar joint. Clin Orthop Relat Res 1970; 68: 124-9.

[12] Ruby LK, Ferenz CC, Dell PC. The pronator quadratus interposition transfer: an adjunct to resection arthroplasty of the distal radioulnar joint. J Hand Surg Am 1996; 21(1): 60-5.

[13] Hotchkiss RN, An KN, Sowa DT, Basta S, Weiland AJ. An anatomic and mechanical study of the interosseous membrane of the forearm: pathomechanics of the proximal migration of the radius. J Hand Surg Am 1989; 14(2): 256-61.

[14] af Ekenstam FW, Palmer AK, Glisson RR. The load on the radius and ulna in different positions of the wrist and forearm. A cadaver study. Acta Orthop Scand 1984; 55: 363-5.

[15] af Ekenstam F, Hagert CG. Anatomical studies on the geometry and stability of the distal radio ulnar joint. Scand J Plast Reconstr Surg 1985; 19: 17-25.

[16] Reid DC. Sport injury asessment \& rehabilitation. New York: Churchill Livingstone 1992; pp 1083-4.

[17] Werner FW, Glisson RR, Murphy DJ, Palmer AK. Force transmission through the distal radioulnar carpal joint: effect of ulnar lengthening and shortening. Handchir Mikrochir Plast Chir 1986; 18(5): 304-8.

[18] Friedman SL, Palmer AK. The ulnar impaction syndrome. Hand Clin 1991; 7(2): 295-310.

[19] Hoag C. Resection of the distal end of the ulna for shortening the radius following fracture. Cal State J Med 1923;21(1): 1-3.

[20] Milch H. Cuff resection of the ulna for malunited Colles' fracture. J Bone Joint Surg Am 1941; 23(2): 311-3.

[21] McBeath R, Katolik LI, Shin EK. Ulnar shortening osteotomy for ulnar impaction syndrome. J Hand Surg Am 2013; 38(2): 379-81.

[22] Tatebe M, Shinohara T, Okui N, Yamamoto M, Hirata H, Imaeda T. Clinical, radiographic, and arthroscopic outcomes after ulnar shortening osteotomy: a long-term follow-up study. J Hand Surg Am 2012; 37(12): 2468-74.

[23] Constantine KJ, Tomaino MM, Herndon JH, Sotereanos DG. Comparison of ulnar shortening osteotomy and the wafer resection procedure as treatment for ulnar impaction syndrome. J Hand Surg Am 2000; 25(1): 55-60.

[24] Nishiwaki M, Nakamura T, Nagura T, Toyama Y, Ikegami H. Ulnar-shortening effect on distal radioulnar joint pressure: a biomechanical study. J Hand Surg Am 2008; 33(2): 198-205.

[25] Arimitsu S, Moritomo H, Kitamura T, et al. The stabilizing effect of the distal interosseous membrane on the distal radioulnar joint in an ulnar shortening procedure: a biomechanical study. J Bone Joint Surg Am 2011; 93(21): 2022-30.

[26] Firoozbakhsh K, Moneim MS, Mikola E, Haltom S. Heat generation during ulnar osteotomy with microsagittal saw blades. Iowa Orthop J 2003; 23: 46-50.

[27] Van Sanden S, De Smet L. Ulnar shortening after failed arthroscopic treatment of triangular fibrocartilage complex tears. Chir Main 2001; 20(5): 332-6.

[28] Tränkle M, van Schoonhoven J, Krimmer H, Lanz U. Indication and results of ulna shortening osteotomy in ulnocarpal wrist joint pain. Unfallchirurg 2000; 103(3): 197-202.

[29] Moermans A, Degreef I, De Smet L. Ulnar shortening osteotomy for ulnar ideopathic impaction syndrome. Scand J Plast Reconstr Surg Hand Surg 2007; 41(6): 310-4.

[30] Rayhack JM, Gasser SI, Latta LL, Ouellette EA, Milne EL. Precision oblique osteotomy for shortening of the ulna. J Hand Surg Am 1993; 18(5): 908-18. 
[31] Chen NC, Wolfe SW. Ulna shortening osteotomy using a compression device. J Hand Surg Am 2003; 28(1): 88-93.

[32] Kitzinger HB, Karle B, Löw S, Krimmer H. Ulnar shortening osteotomy with a premounted sliding-hole plate. Ann Plast Surg 2007; 58(6): 636-9.

[33] Ashan ZS, Song Y, Yao J. Outcomes of ulnar shortening osteotomy fixed with a dynamic compression system. J Hand Surg Am 2013; 38(8): 1520-3

[34] Boardman MJ, Imbriglia JE. Surgical management of ulnocarpal impaction syndrome. J Hand Surg Am 2010; 35(4): 640-51.

[35] Chen F, Osterman AL, Mahony K. Smoking and bony union after ulna-shortening osteotomy. Am J Orthop 2001; 30(6): 486-9.

[36] Wehbé MA, Mawr B, Cautilli DA. Ulnar shortening using the AO small distractor. J Hand Surg Am 1995; 20(6): 959-64.

[37] Cantero J. Reestablishment of supination by shortening the cubitus in complications of Pouteau-Colles fractures (new technic). Z Unfallmed Berufskr 1974; 67(2): 135-7.

[38] Tatebe M, Shinohara T, Okui N, Yamamoto M, Imaeda T, Hirata $\mathrm{H}$. Results of ulnar shortening osteotomy for ulnocarpal abutment after malunited distal radius fracture. Acta Orthop Belg 2012; 78(6): 714-8.

[39] Hollevoet N, Verdonk R, Van Maele G. The influence of articular morphology on non-traumatic degenerative changes of the distal radioulnar joint. A radiographic study. J Hand Surg Br 2006; 31(2): 221-5.

[40] Köppel M, Hargreaves IC, Herbert TJ. Ulnar shortening osteotomy for ulnar carpal instability and ulnar carpal impaction. J Hand Surg 1997; 22-B: 451-6.

[41] Palmer AK, Glisson RR, Werner FW. Relationship between ulnar variance and triangular fibrocartilage complex thickness. J Hand Surg Am 1984; 9(5): 681-2.

[42] Lautenbach M, Millrose M, Schmidt NS, Zach A, Eichenauer F, Eisenschenk A. Ulnocarpal impaction syndrome: treatment with a transverse ulnar shortening osteotomy from an ulnodorsal approach. Arch Orthop Trauma Surg 2014; 134(6): 881-5.

[43] Clark SM, Geissler WB. Results of ulnar shortening osteotomy with a new plate compression system. Hand (NY) 2012; 7(3): 2815 .

[44] Isaacs J, Howard SB, Gulkin D. A prospective study on the initial results of a low profile ulna shortening osteotomy system. Hand (NY) 2010; 5(2): 148-54.

[45] Schmidle G, Arora R, Gabl M. Ulnar shortening with the ulna osteotomy locking plate. Oper Orthop Traumatol 2012; 24(3): 284-92.

[46] Darlis NA, Ferraz IC, Kaufmann RW, Sotereanos DG. Step-cut ulnarshortening osteotomy. J Hand Surg Am 2005; 30(5): 943-8.

[47] Henry M. Subcapital ulnar shorthening osteotomy. J Hand Microsurg 2009; 1(2): 103-7.

[48] Nunez FA Jr, Barnwell J, Li Z, Fiesky A, Nunez FA Sr. Metaphyseal ulnar shortening osteotomy for the treatment of ulnocarpal abutment syndrome using distal ulna hook plate: case series. J Hand Surg Am 2012; 37(8): 1574-9.

[49] Baek GH, Chung MS, Lee YH, Gong HS, Lee S, Kim HH. Ulnar shortening osteotomy in idiopathic ulnar impaction syndrome. Surgical technique. J Bone Joint Surg Am 2006; 88(Suppl 1: Pt 2): $212-20$.

[50] Koh KH, Lee HL, Chang YS, Park MJ. Arthroscopy during ulnar shortening for idiopathic ulnar impaction syndrome. Orthopedics 2013; 36(12): e1495-500.
[51] Kim BS, Song HS. A comparison of ulnar shortening osteotomy alone versus combined arthroscopic triangular fibrocartilage complex debridement and ulnar shortening osteotomy for ulnar impaction syndrome. Clin Orthop Surg 2011; 3(3): 184-90.

[52] Feldon P, Terrono AL, Belsky MR. The "wafer" procedure: partial distal ulnar resection. Clin Orthop Relat Res 1992; 275: 124-9.

[53] Tomaino MM. Results of the wafer procedure for ulnar impaction syndrome in the ulnar negative and neutral wrist. J Hand Surg Br 1999; 24(6): 671-5.

[54] Schuurman AH, Bos KE. The ulno-carpal abutment syndrome: follow-up of the wafer procedure. J Hand Surg Br 1995; 20(2): 171-7.

[55] Loftus JB. Arthroscopic wafer for ulnar impaction syndrome. Tech Hand Upper Extrem Surg 2000; 4(3): 182-8.

[56] Osterman AL. Arthroscopic debridement of triangular fibrocartialge complex tears. Arthroscopy 1990; 6(2): 120-4.

[57] Colantoni J, Chadderdon C, Gaston RG. Arthroscopic wafer procedure for ulnar impaction syndrome. Arthrosc Tech 2014;3(1): e123-5.

[58] Tomaino MM, Weiser RW. Combined arthroscopic TFCC debridement and wafer resection of the distal ulna in wrists with triangular fibrocartilage complex tears and positive ulnar variance. J Hand Surg Am 2001; 26(6): 1047-52.

[59] Slade JF $3^{\text {rd }}$, Gillon TJ. Osteochondral shortening osteotomy for the treatment of ulnar impaction syndrome: a new technique. Tech Hand Up Extrem Surg 2007; 11(1): 74-82.

[60] Greenberg JA, Werner FW, Smith JM. Biomechanical analysis of the distal metaphyseal ulnar shortening osteotomy. J Hand Surg Am 2013; 38(10): 1919-24.

[61] Darrach W. Partial excision of lower shaft of ulna for deformity following Colles's fracture. Ann Surg. 1913; 57: 764-5.

[62] Grawe B, Heincelman C, Stern P. Functional results of the Darrach procedure: a long-term outcome study. J Hand Surg Am 2012; 37(12): 2475-80. e1-2.

[63] Kleinman WB. Salvage procedures for the distal end of the ulna: there is no magic. Am J Orthop (Belle Mead NJ) 2009; 38(4):17280 .

[64] Sauvé L, Kapandji M. Nouvelle technique de traitement chirurgical des luxations récidivantes isolées de l'extrémité inférieure du cubitus. J Chir (Paris) 1936; 47: 589-94.

[65] Shors HC, Baratz ME. Sauvé-Kapandji procedure after distal radius fractures. In: Slutsky DJ, Osterman AL, Eds. Fractures and injuries of the distal radius and carpus: the cutting edge. Saunders, an imprint of Elsevier Inc 2009.

[66] Carter PB, Stuart PR. The Sauvé-Kapandji procedure for posttraumatic disorders of the distal radio-ulnar joint. J Bone Joint Surg Br 2000; 82(7): 1013-8.

[67] George MS, Kiefhaber TR, Stern PJ. The Sauvé-Kapandji procedure and the Darrach procedure for the distal radioulnar joint dysfunction after Colles' fracture. J Hand Surg Br 2004; 29(6): 608-13.

[68] Jacobsen TW, Leicht P. The Sauvé-Kapandji procedure for posttraumatic disorders of the distal radioulnar joint. Acta Orthop Belg 2004; 70: 226-30.

[69] Bowers WH. Distal radioulnar joint arthroplasty: current concepts. Clin Orthop Relat Res 1992; (275): 104-9.

[70] Fernandez DL. Radial osteotomy and Bowers arthroplasty for malunited fractures of the distal end of the radius. J Bone Joint Surg Am 1988; 70(10): 1538-51. 